Research Article

\title{
Histological Changes of the Mandibular Condyle in the Human Fetus at Early Stages of Gestation
}

\author{
Wilfredo Molina \\ Department of Physiology, Faculty of Medicine, University of the Andes Venezuela, Apart ado 109, Merida 5101-A, Venezuela \\ Correspondence should be addressed to Wilfredo Molina; wjmw55@hotmail.com
}

Received 20 October 2013; Revised 30 December 2013; Accepted 7 January 2014; Published 23 February 2014

Academic Editor: Tsutomu Nohno

Copyright (C) 2014 Wilfredo Molina. This is an open access article distributed under the Creative Commons Attribution License, which permits unrestricted use, distribution, and reproduction in any medium, provided the original work is properly cited.

\begin{abstract}
Histochemical studies on the mandibular condyle of the human fetus at gestational ages 12, 14, and 16 weeks were performed. Methods. Histological sections were stained with Schiff's periodic reaction for glicoproteins, hematoxiline eosine detects mesenchymal tissue and trichhromic stain for collagen. The ANOVA one-way test was used to evaluate the differences during stained zones in the three fetus groups. Results. The percentage of glycoproteins and mesenchymal tissue was denser at 12 weeks. This percentage decreases at 14 weeks and is less at 16 weeks. An increase in the amount of collagen in the studied weeks was observed. The percentages of glycoproteins, mesenchymal tissue, and collagen were significantly different; $f=4373,9624.8$, and $3674, P<0.0001$ for the three studied groups. Conclusion. The endochondral bone formation of the mandibular condyle includes modifications of the quantities of glycoproteins, mesenchymal tissue, and collagen.
\end{abstract}

\section{Introduction}

During development of the human fetus, the secondary cartilage appears later in the mandibular condyle in the embryonic stage, and this cartilage is independent of primary skeletal cartilage. The two types of cartilages show differences in histological organization and in the pattern of cell proliferation [1]. The mandibular condylar cartilage is often classified as secondary cartilage because it differs to some extent from primary skeletal cartilage [2]. This cartilage appeared to have maintained its inherent structural characteristics, including all its various layers: chondroprogenitor, chondroblastic, and hypertrophic chondrocytes. The mandibular condylar cartilage (MCC) grows strictly in an appositional mode; mesenchymal progenitor cells differentiate to functional chondroblast, which subsequently mature to hypertrophic chondroblast [3].

The condylar cartilage appears to acquire its shape during week 11 of growth. During development period considered, the MCC consists of five cell layers: the articular layer, mesenchymal layer, chondroblastic layer, the chondrocytes layer, and hypertrophic chondrocytes [4]. Three phases have been identified in temporomandibular joint development (TMJ): (1) the blastemic stage (weeks 7-8 of development);
(2) the cavitations' stage (weeks 9-11 of development); and (3) the maturation stage (after weeks 12 of development). The chondrification of mandibular condyle begins in week 9 of development [5].

A prechondrogenic mesenchymal cell probably promotes the formation of cartilage by stimulating an expansion of the chondroprogenitor population [6]. The condylar cartilage in the mandible is formed from ectomesenchyme of the neural crest. In the mandibular condyle, the stem cells that give rise to skeletoblast are produced at the expense of mesenchymal tissue. The skeletoblasts spontaneously differentiate into osteoblasts, and only under influence of certain environmental conditions they differentiate into prechondroblasts [7].

In the developing fetus, the region between the cartilage of the mandibular condyle and the articular surface of the developing temporal bone is filled with ectomesenchyme witch contains mesenchymal cells. These cells suffer differentiation to form osteoblasts. At ten weeks of gestation, the first mesenchymal differentiation on the cartilage of the mandibular condyle begins. The mandible is formed for membranous ossification of the mesenchymal tissue. During the interaction between epithelial and mesenchymal tissues, the mesenchymal cells form odontoblast [8]. 
In the mandibular condyle, the chondrocytes maturation is started with mesenchymal differentiation into prechondroblasts and ended with highly matured hypertrophic chondrocytes. This mesenchymal tissue covering of the condyle is responsible for a fundamental characteristic of the condylar cartilage. There are mesenchymal cells first, and these cells differentiated into cartilage as a secondary event [9].

During the mandibular condylar growth the presence of some types of proteins seems crucial for developmental process and especially in control of the mesenchymal Cells into chondrocytes. High levels of mesenchymal condensation in areas of mandibular condyle before chondrocytes differentiation have been found. Some kinds of proteins as sox9 have been also associated with regulation process of the chondrocytes to synthesize collagen in condylar cartilage [10]. The other important factor in the growth of the human mandibles is the glycoprotein; the cell surface glycoprotein is involved in the control of cell maturation, differentiation and cell adherence [11]. After maturation, the chondrocytes progress toward hypertrophy and secrete type $\mathrm{x}$ collagen which indicates the termination of chondrogenesis [12]. Type $\mathrm{x}$ collagen is the matrix for the hypertrophic cartilage destined for endochondral ossification [13].

The developmental sequence of the bone cell differentiation is characterized by three principal periods: proliferation, extracellular maturation, and mineralization [14]. During the period of proliferation and cell differentiation, mesenchymal cells proliferate and some become osteoblasts. Osteoblasts secrete osteoid collagen-proteoglycan, with great ability to bind calcium. These processes of intramenbranous ossification involve bone morphogenetic proteins and transcription factors [15].

The initiators of the process of mineralization are small, round, extracellular, lipid, bilaminar organelles that bud from hypertrophic chondrocytes or osteoblasts. During this process, collagen fibrils, fibronectin, and glycoprotein determine the orientation and organization of the bone mineral crystal. Other glycoproteins such as glycosaminoglycans appear to play a role in the inhibition of excessive mineralization [16]. In weeks ten and 11 of development, the vascular channels appear in the condylar cartilage and intramembranous ossification process begins [17]. In the same way, since week 14 of fertilization, the growth of conical structure in the condylar blastema has been observed on histological studies. The presence of this growth center for the trabecular bone of mandibular body indicates that the initial growth process of the endochondral ossification of the condyle has already begun [18].

The early stages of formation of the future condyle are characterized by condensation of mesenchymal cells and abundant noncollagen protein. In later stages also the presence of large amounts of collagen type I is observed. Collagen, and proteoglycan synthesis have been used as indicators of proliferation and differentiation of condylar chondrocytes [19]. The chondrocytes exit the proliferative cycle, hypertrophy, and produce collagen Type $\mathrm{x}$. This collagen is reabsorbed and is replaced by bone [20].
The mandibular condyle is involved in endochondral bone formation [21]. It has been showed that chondrocytes proliferation of condylar cartilage is modulated by mechanical factors associated with mandible and temporomandibular junction (TMJ) movement [22]. In the human fetuses, the mandible lips and tongue movements were observed at early stage of intrauterine life [23]. In the same way, the prenatal jaw movement has been associated as important factor in bone formation of the mandibular condyle [24].

In this study the development of the mandibular condylar cartilage in the human fetuses was observed, with the aim of evaluating some histological aspects, which may have a bearing on the growth of the condylar cartilage between 12 and 16 weeks of prenatal period. The studied aspects were the quantities of mesenchymal tissue, glycoprotein, and collagen in the mandibular condyle in these stages of gestation.

\section{Materials and Methods}

2.1. Surgical Procedures. The surgical procedures were previously clarified. The temporomandibular joints (TMJs) of 18 human fetuses aborted at 12,14, and 16 weeks of gestation were removed in $1 \mathrm{~cm}^{3}$. The skin tissue of the selected temporomandibular joints was removed. These blocks were separated and identified according to gestational age.

2.2. Study Groups. The fetuses were divided into three groups of 12,14 , and 16 weeks of gestation ( 6 fetuses of each age) and the histological sections of each fetus group were subdivided into glycoprotein, mesenchymal tissue, and collagen, respectively.

2.3. Histological Preparation. Condyle of the mandibles of human fetuses aborted at 12, 14, and 16 gestation was removed in blocks. These blocks were fixed in 10\% stabilized neutral formalin containing $40 \%$ formaldehyde, $4.0 \mathrm{~g}$ of sodium dehydrogenate phosphate, and $6.5 \mathrm{~g}$ of disodium hydrogen phosphate at $\mathrm{Ph} 7.0$ and stored at $4^{\circ} \mathrm{c}$. The blocks were embedded in paraffin, and these paraffin-embedded blocks were sectioned serially in sagittal planes $6 \mu \mathrm{m}$ wide with a rotatory microtome. Fifth teen sections were obtained for each mandible condyle. Sections were deparaffinized and hydrated in distilled water. Schiff's periodic acid reaction (pas), trichromic stain, and hematoxiline eosine were used for glycoprotein, collagen, and mesenchymal tissue, respectively. The protocol used was the US Armed Force Institute of Pathology histotechnology method [25]. Each section was cut and mounted on a previously numbered glass slide and independently assessed by two members of the histological department of the Medicine School of the University of Los Andes, Venezuela; all of the observers were specialist in histology. There was no calibration with respect to their rating, but the two observer's reports were similar. The selected sections were photographed and digitalized.

2.4. Quantitative Analysis. Quantitative analysis was performed with serial sections for each temporomandibular joint. The total amount of glycoprotein, mesenchymal tissue, 
and collagen was calculated for each histological section by ImageJ software. This method was used to analyze the percentage of stained and unstained zones of glycoprotein, collagen, and mesenchymal tissue. In all the selected cases, the average of the percentage of stained and unstained areas of the mandibular condyle was calculated. All the images were obtained through a transmitted light microscope (Kyowa Medilux-12) with video camera (motic 2000) and motic images 2.0 multilanguge application software for the acquisition of the differences in staining properties and morphology facilitating identification of all studied tissues.

2.5. Classification of the Results of Histological Sections. The results were classified into four types as follows:

(1) type I: absence of glycoprotein, collagen, or mesenchymal tissue;

(2) type II: high quantity of glycoprotein, collagen or mesenchymal tissue;

(3) type III: isolated areas of glycoprotein, collagen, or mesenchymal tissue;

(4) type IV: less quantities of glycoprotein, collagen, or mesenchymal tissue.

2.6. Statistical Methods. The ANOVA one-way test was used to evaluate the differences among stained areas.

No fetus used in this study had any visible evidence of developmental abnormalities or genetic disorders. To carry out this study, samples of aborted human fetuses were used in accordance with procedures approved by the department of the physiology of the Medicine School of the University of Los Andes. All the conditions of Helsinki declaration related to research in diseased human subjects were observed.

\section{Results}

3.1. Glycoprotein. The histological sections revealed the presence of glycoprotein in all layers of the mandibular condyle at 12 weeks of gestation. This distribution was uniform among osteoprogenitor cells and the bone formative zone. The stained areas showing the presence of glycoprotein in the anterior, middle, and posterior zones of the condyle (Figure 1) and the chondrocytes are surrounded by a glycoprotein matrix. Therefore, in any histological sections this matrix of glycoprotein was found in isolated areas of the mandible condyle. All 12-week-old fetuses were classified type II.

At 14 weeks, dark pink areas with pas stain for glycoprotein were distributed in the upper zone of the condyle and have been observed occupying the anterior, middle, and posterior areas in the resting and proliferative layers between osteoprogenitor cells. Because the erosive zone has advanced to the head of the condyle. The distribution of glycoprotein was uniform in the stained area. All six 14-week-old fetuses were classified type III (Figure 2). In the six 16-week-old fetuses, the glycoprotein was localized in a less area of the upper and posterior zones of the condyle. Unstained areas were observed in the anterior zone. Osteoprogenitor cells

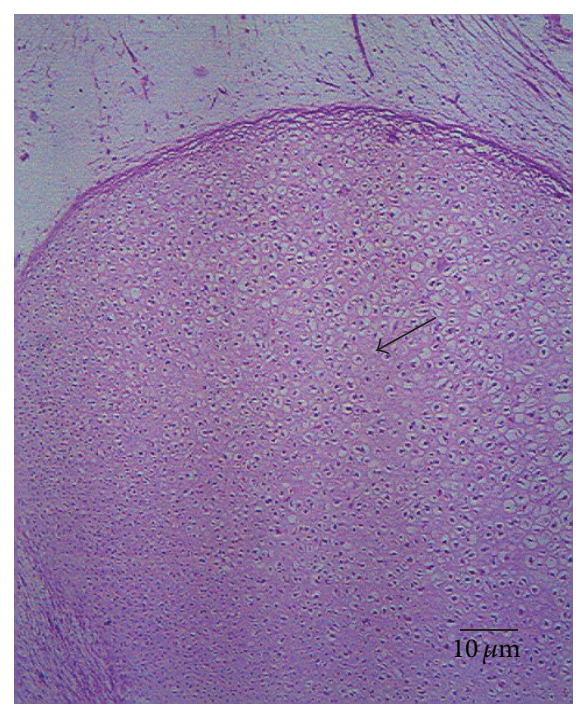

Figure 1: Mandible condyle of human fetus. At 12 weeks of gestation the glycoprotein fills large surface in all zones of the condylar head.

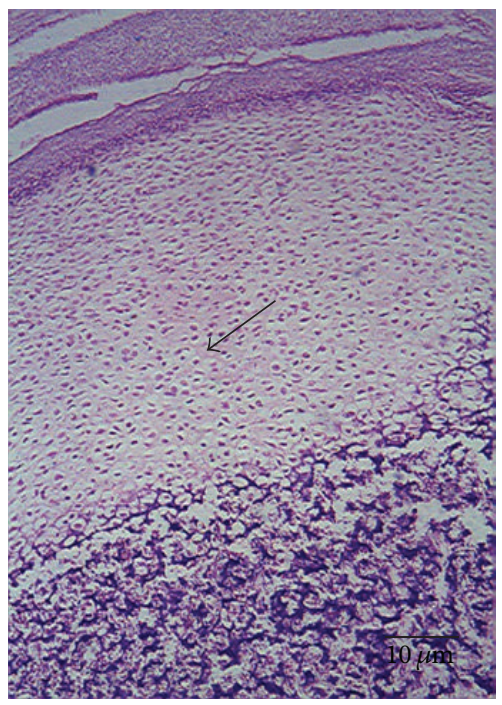

FIGURE 2: Fetus at the age of 14 weeks. The glycoprotein occupies the upper pink zone of the condyle. Empty spaces can be observed without glycoprotein in the extracellular matrix between osteoprogenitor cells.

occupy almost all of the upper area of the condylar head (Figure 3).

3.2. Mesenchymal Tissue. Articular, resting, and proliferative layers were observed mainly composed by mesenchymal cells at 12 weeks of intrauterine life. These mesenchymal cells were found occupying larges areas in the anterior, middle, and posterior zones of the condyle (Figure 4 ) and were considered type II.

All six 14-week-old fetuses were classified type III. The mesenchymal tissue was observed in small areas in upper and posterior zones of the condyle, close to the hypertrophic zone, 


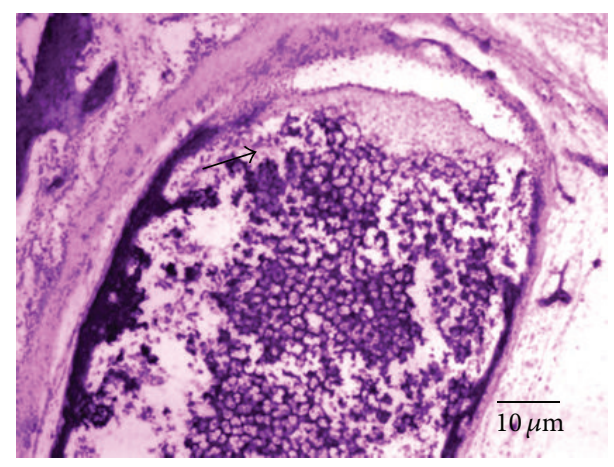

FIgURE 3: Mandibular condyle of human fetus at 16 weeks of gestation. The arrow shows a small quantity of glycoprotein occupying smaller proportion in the upper and posterior zone of the condyle. A greater amount can be observed in the articular area.

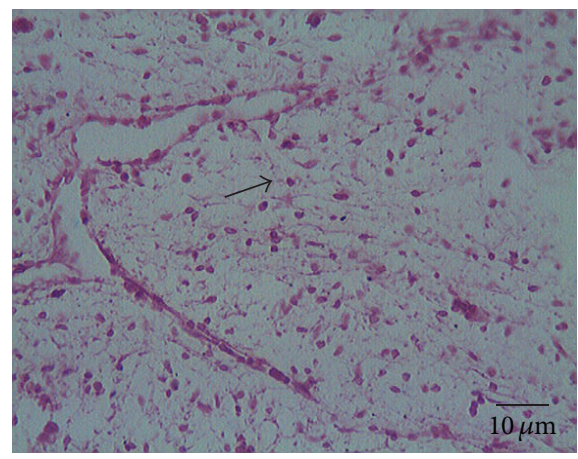

Figure 4: Condyle of human fetus of 12 weeks of gestation. Arrow shows the mesenchymal tissue occupying large areas in the condylar head.

which were the major advances on the back of the condylar head (Figure 5).

In the six 16-week-old fetuses, the mesenchymal tissue was localized in less zone among osseous cells and in this stage of gestation, the mesenchymal tissues have been replaced by osseous matrix and represent a smaller proportion in the posterior zone of the condyle (Figure 6).

3.3. Growth Cone in the Mandibular Condyle. The growth cone was observed at 12 weeks of gestation. The vertex of the cone was located in the lower areas of the head of the condyle in formation and the base to the condylar neck (Figure 7). Osteoblasts in band disposition at 12 weeks, the osseous matrix osteoid at 14 weeks, and the osteocyte with cytoplasm extensions at 16 weeks were found (Figures 8,9 , and 10, resp.). Both osteoblasts and osteocyte are surrounded by an osteoid matrix. These cells are oriented in the growth direction from the neck area in the erosive and proliferative zones towards the head of the condyle. The process of endochondral ossification and matrix calcification in the histological sections studied follows a direction from the base to the apex of the growth cone. At 14 weeks, histomorphological images obtained show the progress of the newly formed bone area. Erosive and proliferative zones were observed without precise

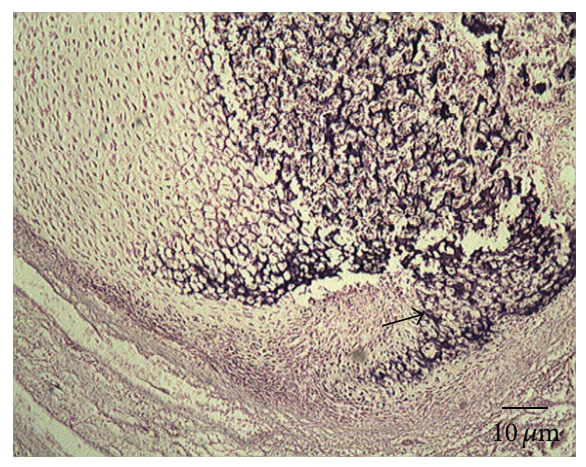

FIGURE 5: 14 weeks of intrauterine life. The arrow shows a smaller quantity of the mesenchymal tissue in the posterior zone of the condyle. There were not observed mesenchymal cells in other regions of the condyle in these histological sections.

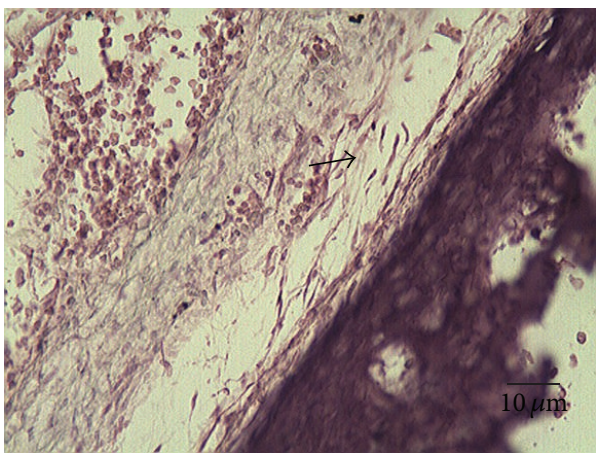

FIgURE 6: The mesenchymal tissue is located in fewer areas in the posterior zone of the condyle at 16 weeks of gestation. The arrow shows mesenchymal cells in the posterior edge of the condyle.

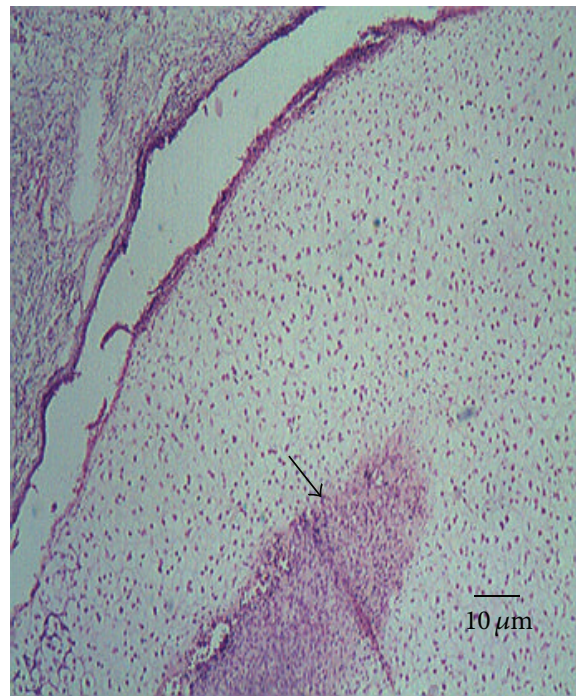

FIGURE 7: The arrow shows the growth cone its location is toward the bottom, middle, and the center of the condyle; it has not yet attained the upper portion of the condylar head. Its base is toward the neck and the vertex is directed to the articular area at 12 weeks. 


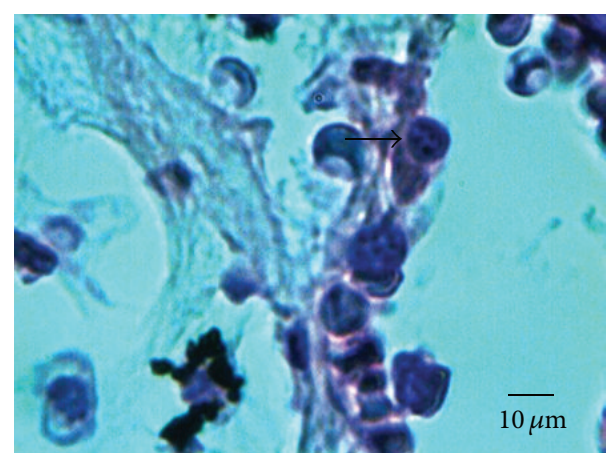

FIGURE 8: Osteoblasts in band disposition can be observed at 12 weeks of gestation.

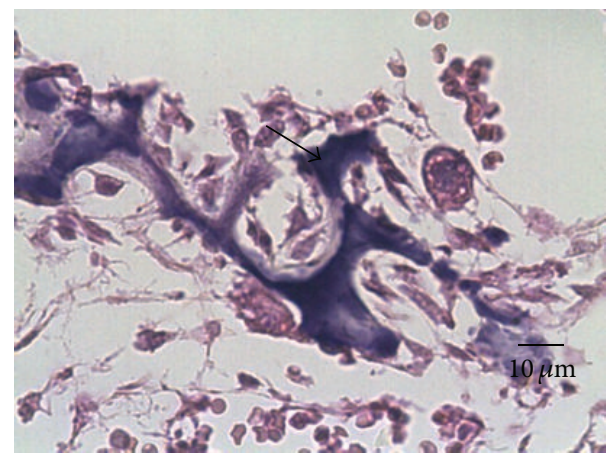

FIGURE 9: Osseous matrix osteoid and ossiform in the human fetus at 14 weeks.

edges for bone penetration. The process of penetration of this bone matrix is observed in the central region of the condylar head and neck. Osteoid tissue was not observed in the anterior or the posterior areas of the condyle at this stage of gestation (Figure 11).

3.4. Collagen. In the present investigation it was observed that the collagen deposits follow a direction from the condylar neck to the top of the head of the condyle at 12,14, and 16 weeks in the human fetus (Figures 12, 13, and 14). Collagen synthesis was found with a significant increase in histological sections from 12 to 16 weeks of intrauterine life. A decrease for collagen in gestational stages studied was not observed. The results indicate that the amount of collagen located in stained areas of the condylar head present significant differences compared with other areas at 12 weeks. Moreover, areas of collagen were clearly identified between osteoprogenitor cells only in the lower portion of the condyle. The histological sections corresponding to 14 weeks of gestation showed scattered areas of collagen deposits from the lower to the upper portion of the condylar head with clear differences from the corresponding histological sections at week 16 where the collagen deposited in the head the condyle shows a more compact and uniform appearance.

The densities of the stained zones as measured by ImageJ software were $83.36 \%, 16.93 \%$, and 4.18 for glycoprotein, 8.34 , 34.96 , and 59.49 for collagen matrix, and 84.12, 31.34, and 3.37

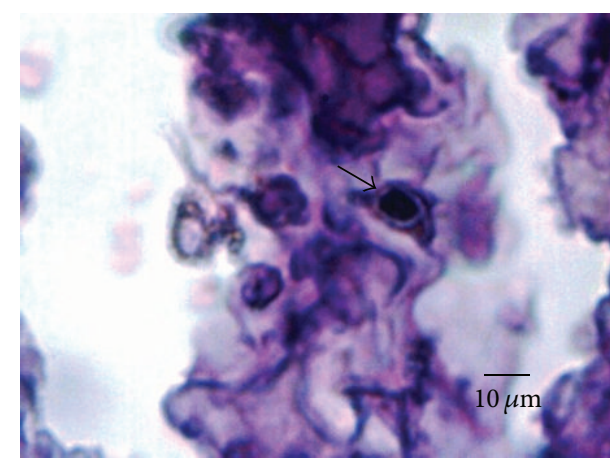

FIGURE 10: Osteocyte with cytoplasm extensions inside matrix of human fetus at 16 weeks.

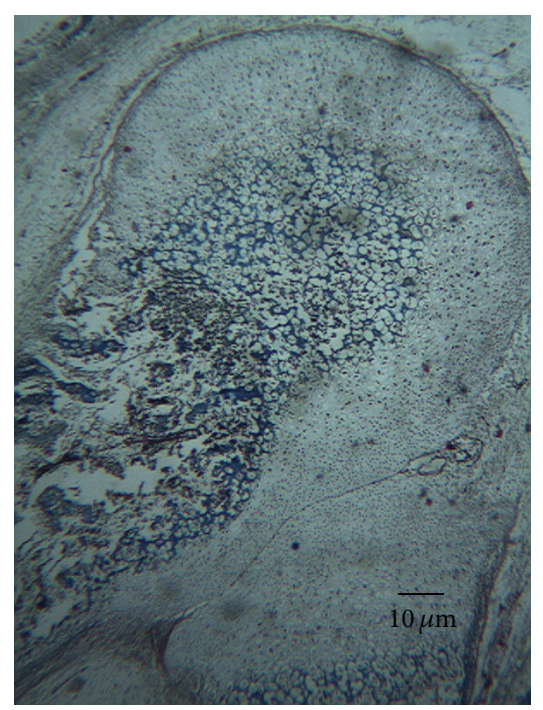

FIGURE 11: The bone matrix formation is observed in the neck to the head of the mandibular condyle in the central areas at 12 weeks of prenatal period. Osteoid tissue and the erosive areas were observed in the lower portion of the neck of the condyle.

for mesenchymal tissue at 12, 14, and 16 weeks of gestation, respectively.

In the studied cases, glycoprotein, collagen, and mesenchymal tissue and the ANOVA one-way test showed that the differences among the selected groups were statistically significant: $f=4373.28$ for glycoprotein, 9624.8 for mesenchymal tissue, 3674 for collagen, and $P<0.0001$ for the three fetus groups.

\section{Discussion}

The development of the mandibular condyle could be involved by many factors. Disturbances in prenatal temporomandibular joint movements can be considered on developmental hypoplasia of the mandible condyle [26]. The mandible is one earliest bone to undergo ossification [27]. This study showed that the histological differentiation on the mandibular condyle has already begun by the 12 weeks 


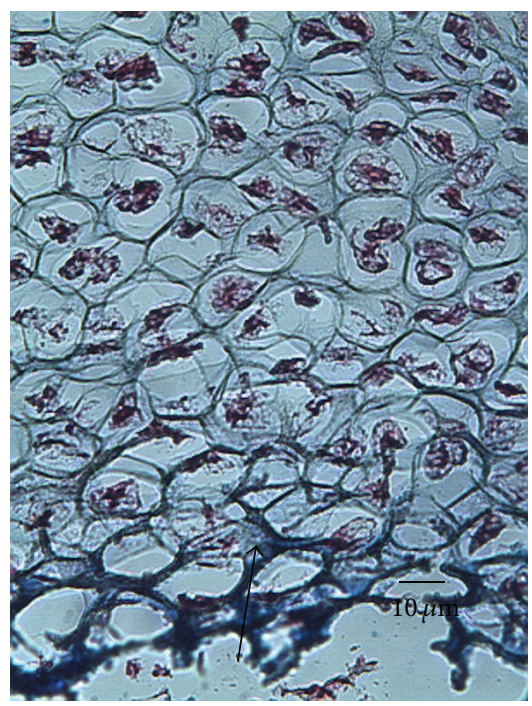

FIGURE 12: Collagen deposited in the lower portion of the mandibular condyle at 12 weeks of gestation can be observed. Deposits are not observed toward the articular area.

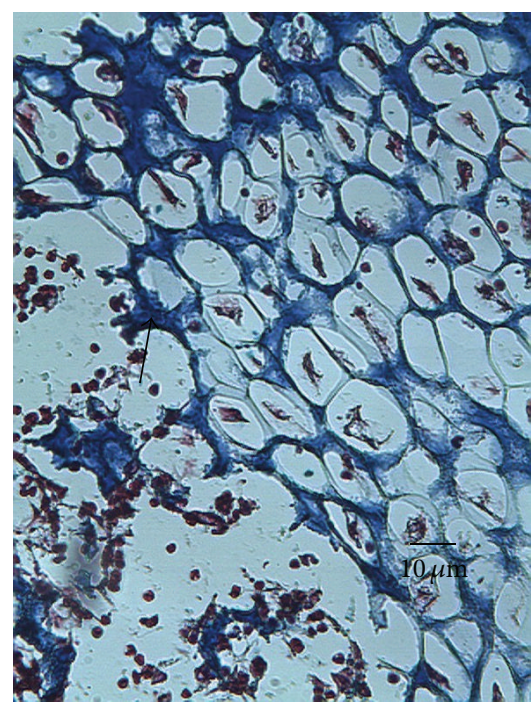

FIgURE 13: The arrow shows the collagen deposited in the lower and middle portion between osteoprogenitor cells of the mandibular condyle at 14 weeks of gestation.

of intrauterine life. The condyle was recognized as a mesenchymal condensation at 14.5 days and the mesenchymal condensation of the condyle had differentiated into chondrocytes by 16.5 days in rats fetuses [28]. It was noted in this investigation that the tissue occupies large areas of the surface at 12 weeks of pregnancy; this mesenchymal tissue is replaced by matrix of osteoprogenitor cells on the posterior stage of gestation. At 16 weeks a smaller mesenchymal zone located in the upper area of the condyle was found. In studies at early stage of development in cultures it has been mentioned that mesenchymal cells condense to form a core of differentiated chondrocytes; osteogenesis starts at the periphery of the

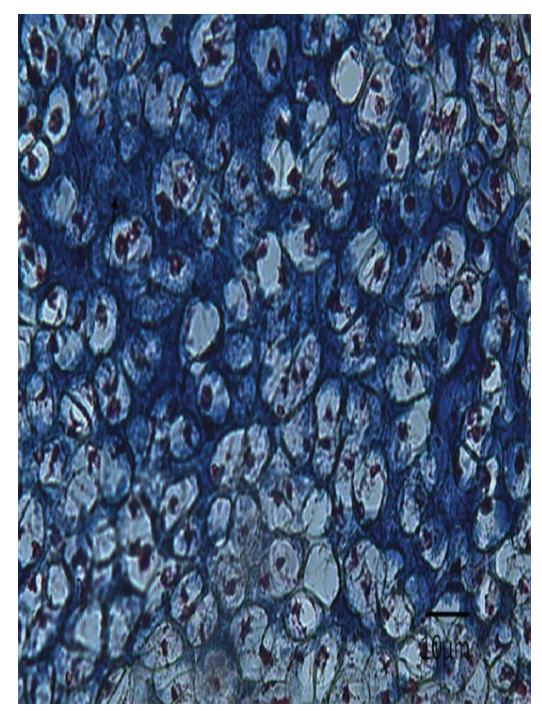

FIGURE 14: Collagen seems occupy to the lower, middle, and upper areas between osteoprogenitor cells of the mandibular condyle at 16 weeks.

cartilage core; this is subsequently replaced by trabecular bone.

It has been postulated that growth plate hypertrophic chondrocytes might also contribute to the formation of the bone matrix. The presence of osteoblasts has been associated with the synthesis and mineralization of the osteoid extracellular matrix. The present investigation shows osteoblasts surrounded by osteoid matrix and suggests that in the prenatal period studied here the mineralization process of the mandible condyle has already begun.

Regarding glycoprotein during growing periods it has been located among the anterior, central, and posterior areas of the condyle [29]. Just as the glycoprotein has also shown that substances such as glycosaminoglycans are between the most important elements in the formation of cartilage, these elements are very important to chondrogenesis and therefore to normal skeleton formation [30]. This research showed that the quantities of glycoprotein decreased at 16 weeks and are much less compared with 12 and 14 weeks of intrauterine life. The spaces were occupied by glycoprotein and mesenchymal tissue between the osteoprogenitor cells in different cell layers of the mandibular condyle seem to be filled by collagen. There are correlations among synthesis of type $\mathrm{X}$ collagen and occurrence of endochondral ossification; type $\mathrm{X}$ collagen eases this ossification by regulating matrix mineralization. This type $\mathrm{X}$ collagen is a reliable marker for new bone formation in articular cartilage [31]. In this study, the collagen matrix was observed occupying larger areas among osteoprogenitor cells at 16 weeks of intrauterine life. Collagen seems to grow not only in the condyle of the mandible but also in other structures such as the articular disc, to the extent that advances human fetal gestation [32]. Immunohistochemical examination showed the presence of collagen type I and type II in the extracellular matrix of the mandible condylar cartilage in rats. These observations 
reveal the presence of chondroid bone, a tissue intermediate between bone and cartilage tissues, in the mandible condylar cartilage, and present the possibility of osteogenic transdifferentiation of mature chondrocytes [33]. However, in the human fetuses, this presence of collagen in the extracellular matrix of the mandible condylar cartilage is only possible in later stage of osteogenesis. In this study, collagen deposits were only located in the upper portion of the erosive layer and isolated areas of hypertrophic zone at 12 weeks and at 16 weeks of the prenatal period when these deposits reach the articular region. The presence of osteoblasts arrangeed in bands, osteoid matrix, and osteocyte with cytoplasm extensions, structures that were found in histological sections at 12,14 , and 16 weeks, respectively, in the areas adjacent to the erosive zone, and how the collagen is deposited in the mandibular condyle during these periods of fetal growth make it possible to suggest that chondrocytes maturation begins in the areas near the erosive zone and the articular surface of the condyle is the last area to mature in the human fetus. The findings in this investigation show that the mesenchymal cells, glycoprotein, and collagen concentrations are modified during chondroprogenitor cells differentiation and the osteogenic formation process at early stage of gestation.

\section{Conclusion}

This investigation showed that glycoprotein and mesenchymal tissue were found occupying a large quantity along the mandibular condyle at 12 weeks of gestation; these quantities decreased during 14 and 16 weeks. In this stage (16 weeks) both glycoprotein and mesenchymal tissue occupying very small areas in the mandibular condyle. The mesenchymal tissue and glycoprotein have been replaced by collagen during the formation of the osteoprogenitor cells. It seems reasonable to suggest that in the mandibular condyle, the collagen is an important element in the bone formation in this stage of gestation.

\section{Conflict of Interests}

The author declares that there is no conflict of interests regarding the publication of this paper.

\section{Acknowledgments}

The author wants to thank Doctors Gisela Sosa (Pathological Anatomy Unit of Valencia Central Hospital), Luis Betancourt (Histology Department of University of Los Andes), and Ana Barrios (Pathological Anatomy Unit of Valencia Central Hospital) for their kind assistance.

\section{References}

[1] M. Delatte, J. W. Von Den Hoff, R. E. M. Van Rheden, and A. M. Kuijpers-Jagtman, "Primary and secondary cartilages of the neonatal rat: the femoral head and the mandibular condyle," European Journal of Oral Sciences, vol. 112, no. 2, pp. 156-162, 2004.
[2] P. Proff, T. Gedrange, R. Franke et al., "Histological and histomorphometric investigation of the condylar cartilage of juvenile pigs after anterior mandibular displacement," Journal of Dental Research, vol. 84, pp. 474-479, 2007.

[3] J. C. Copray, H. W. Jansen, and H. S. Duterloo, "The role of biomechanical factors in mandibular condylar cartilage growth and remodeling in-vitro," in Developmental Aspects of TemporoMandibular Joint Disorders, Monograph 16, Craniofacial Growth Series, University of Michigan, Ann Arbor, Mich, USA, 1985.

[4] B. Thilander, G. E. Carlsson, and B. Ingervall, "Postnatal development of the human temporomandibular joint. I. A histological study," Acta Odontologica Scandinavica, vol. 34, no. 2, pp. 117-126, 1976.

[5] L. Suk, Y. S. Kim, H. S. Oh, K. H. Yang, E. C. Kim, and J. G. Chi, "Prenatal development of the human mandible," Anatomical Record, vol. 265, no. 3, pp. 314-325, 2001.

[6] Y. Hiraki, C. Shukunami, K. Iyama, and H. Mizuta, "Differentiation of chondrogenic precursor cells during the regeneration of articular cartilage," Osteoarthritis and Cartilage, vol. 9, supplement 5, pp. S102-S108, 2001.

[7] A. G. Petrovic, "Postnatal growth of bone: a perspective of current trends, new approaches, and innovations," Progress in Clinical and Biological Research, vol. 101, pp. 297-331, 1982.

[8] S. N. Bhaskar, "Articulacióntemporo-mandibular," in Histología y Embriología Bucal, pp. 446-448, El Ateneo, Buenos Aires, Argentina, 1983.

[9] G. Shen, Active mandibular forward positioning a molecular and biochemical study [Ph.D. thesis], University of Hong Kong, Hong Kong, 2000.

[10] A. B. Rabie, T. T. She, and U. Hägg, "Functional appliance therapy accelerates and enhances condylar growth," American Journal of Orthodontics and Dentofacial Orthopedics, vol. 123, no. 1, pp. 40-48, 2003.

[11] M. Vernay, M. Cornic, M. Aubery, and R. Bourrillon, "Relationship between regeneration of cell surface glycoproteins in trypsin-treated chick embryo fibroblasts and cell adhesion to the substratum," Experientia, vol. 34, no. 6, pp. 736-737, 1978.

[12] G. Shen, A. B. Rabie, Z. Zhao, and K. Kaluarachchi, "Forward deviation of the mandibular condyle enhances endochondral ossification of condylar cartilage indicated by increased expression of type X collagen," Archives of Oral Biology, vol. 51, no. 4, pp. 315-324, 2006.

[13] A. B. M. Rabie, G. Shen, U. Hagg, and T. Kaluarachchi, “Type x collagen-a marker for endochondral ossification of mandibular condyle," in Orthodontics Year Book, pp. 50-58, 2000.

[14] J. B. Lian and G. S. Stein, "Concepts of osteoblast growth and differentiation: basis for modulation of bone cell development and tissue formation," Critical Reviews in Oral Biology and Medicine, vol. 3, no. 3, pp. 269-305, 1992.

[15] B. Hall, “The embryonic development of bone," Scientific American, vol. 76, no. 26, pp. 174-181, 1998.

[16] K. Yagami, J. Suh, M. Enomoto-Iwamoto et al., "Matrix GLA protein is a developmental regulator of chondrocyte mineralization and, when constitutively expressed, blocks endochondral and intramembranous ossification in the limb," Journal of Cell Biology, vol. 147, no. 5, pp. 1097-1108, 1999.

[17] J. R. Mérida Velasco, J. F. Rodríguez Vázquez, C. De La Cuadra Blanco, R. Campos López, M. Sánchez, and J. A. Mérida Velasco, "Development of the mandibular condylar cartilage in human specimens of 10-15 weeks' gestation," Journal of Anatomy, vol. 214, no. 1, pp. 56-64, 2009. 
[18] S. K. Lee, Y. S. Kim, H. S. Oh, K. H. Yang, E. C. Kim, and J. G. Chi, "Prenatal development of human mandible," Anatomical Record, vol. 263, no. 3, pp. 314-325, 2001.

[19] L. G. de Bont, P. de Haan, and G. Boering, "Structure and growth of the cartilage of the temporomandibular joint," Nederlands Tijdschrift voor Tandheelkunde, vol. 92, no. 5, pp. 184-189, 1985.

[20] X. Nie, K. Luukko, and P. Kettunen, "BMP signalling in craniofacial development," International Journal of Developmental Biology, vol. 50, no. 6, pp. 511-521, 2006.

[21] M. Silbermann and J. Frommer, "Heterogeneity among chondrocytes of the mandibular condyle in foetal and postnatal mice," Archives of Oral Biology, vol. 18, no. 12, pp. 1549-1554, 1973.

[22] M. R. Simon, "The role of compressive forces in the normal maturation of the condylar cartilage in the rat," Acta Anatomica, vol. 97, no. 3, pp. 351-360, 1977.

[23] G. Couly, "Les neurocristopathies du bourgeon naso-frontal," Revue de Stomatologie et de Chirurgie Maxillo-Faciale, vol. 82, no. 2, pp. 213-225, 1981.

[24] H. Habib, T. Hatta, J. Udagawa, L. Zhang, Y. Yoshimura, and H. Otani, "Fetal jaw movement affects condylar cartilage development," Journal of Dental Research, vol. 84, no. 5, pp. 474479, 2005.

[25] L. G. Luna, Manual of Histological Staining Methods, Armed Forces Institute of Pathology, New York, NY, USA, 1968.

[26] D. A. Keith, "Development of the human temporomandibular joint," British Journal of Oral Surgery, vol. 20, no. 3, pp. 217-224, 1982.

[27] M. H. Kaufman, The Atlas of Mouse Development, Academy London, London, UK, 2002.

[28] Y. Yamaki, K. Tsuchikawa, T. Nagasawa, and K. Hiroyasu, "Embryological study of the development of the rat temporomandibular joint: highlighting the development of the glenoid fossa," Odontology, vol. 93, no. 1, pp. 30-34, 2005.

[29] I. Takahashi, I. Mizoguchi, Y. Sasano et al., "Age related changes in the localization of glycosaminoglycans in condylar cartilage of the mandible in rats," Anatomy and Embryology, vol. 194, no. 5, pp. 489-500, 1996.

[30] J. Shambaugh and W. A. Elmer, "Analysis of glycosaminoglycans during chondrogenesis of normal and brachypod mouse limb mesenchyme," Journal of Embryology and Experimental Morphology, vol. 56, pp. 225-238, 1980.

[31] G. Shen, "The role of type X collagen in facilitating and regulating endochondral ossification of articular cartilage," Orthodontics \& Craniofacial Research, vol. 8, no. 1, pp. 11-17, 2005.

[32] W. Molina, S. Pino, G. Sosa, and L. Hernández, "Distribution of mucopolysaccharides and glycoproteins in the articular discs of temporomandibular joints in human fetuses," Journal of Orofacial Pain, vol. 19, no. 4, pp. 325-330, 2005.

[33] I. Mizoguchi, M. Nakamura, I. Takahashi, Y. Sasano, M. Kagayama, and H. Mitani, "Presence of chondroid bone on rat mandibular condylar cartilage. An immunohistochemical study," Anatomy and Embryology, vol. 187, no. 1, pp. 9-15, 1993. 

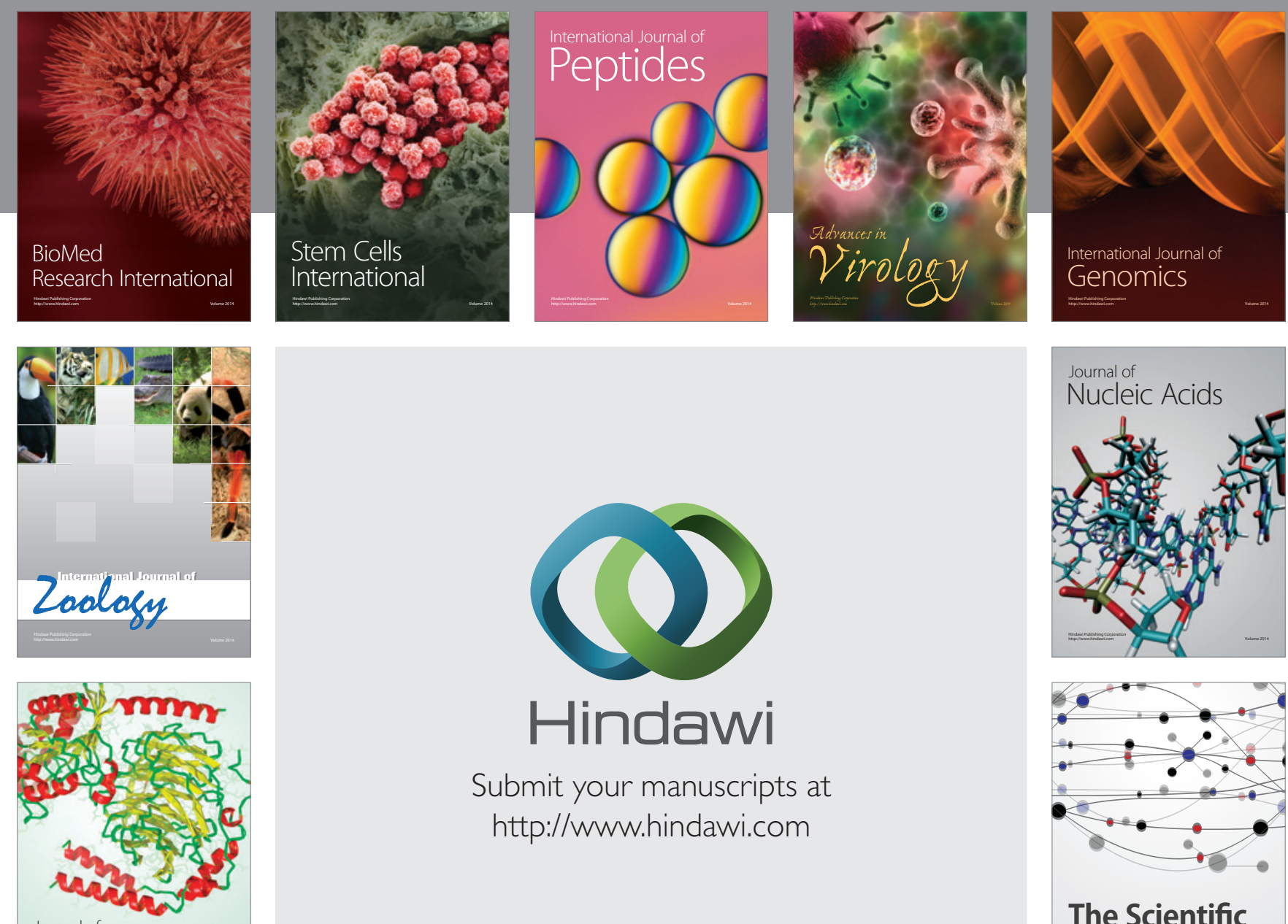

Submit your manuscripts at

http://www.hindawi.com

Journal of
Signal Transduction
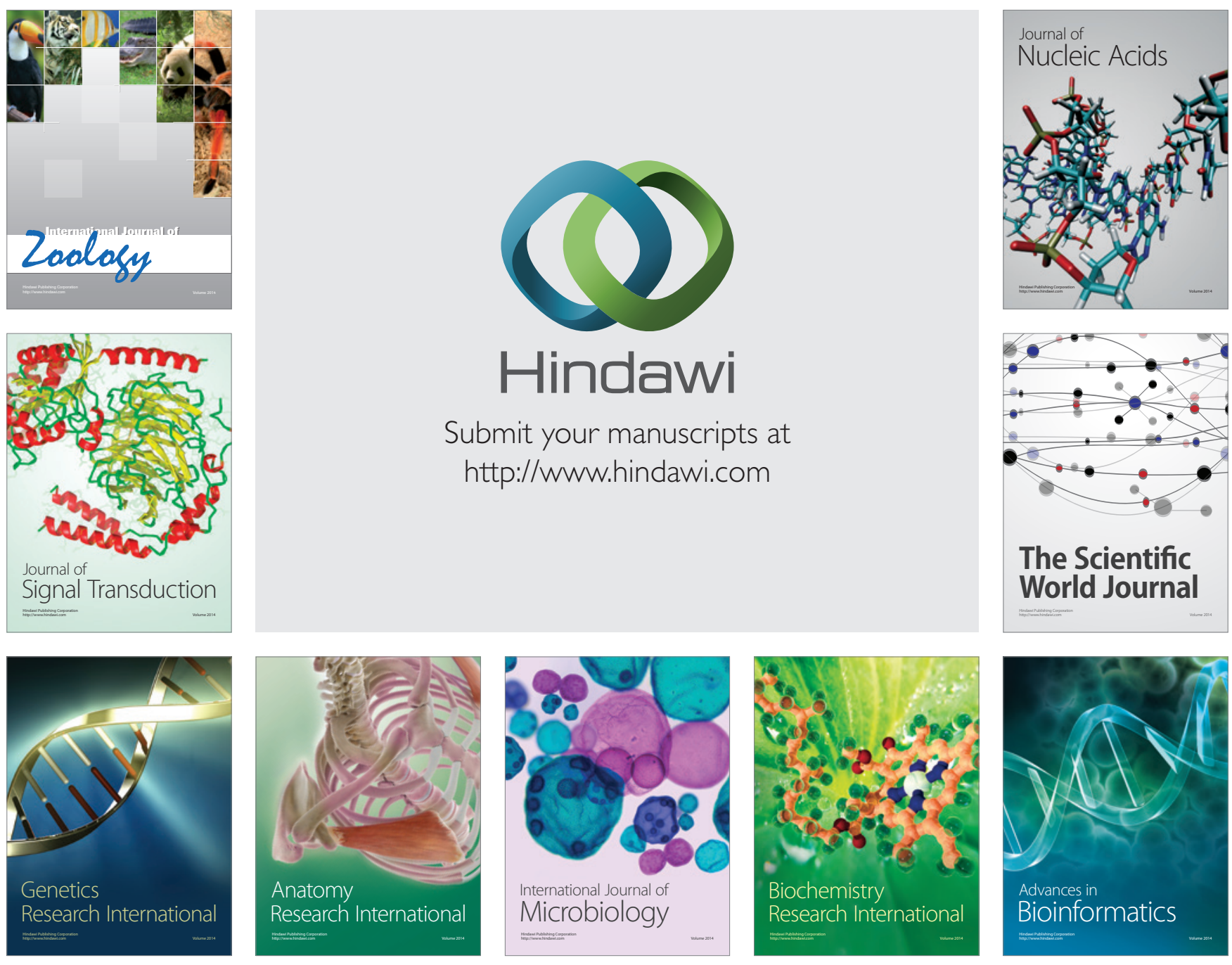

The Scientific World Journal
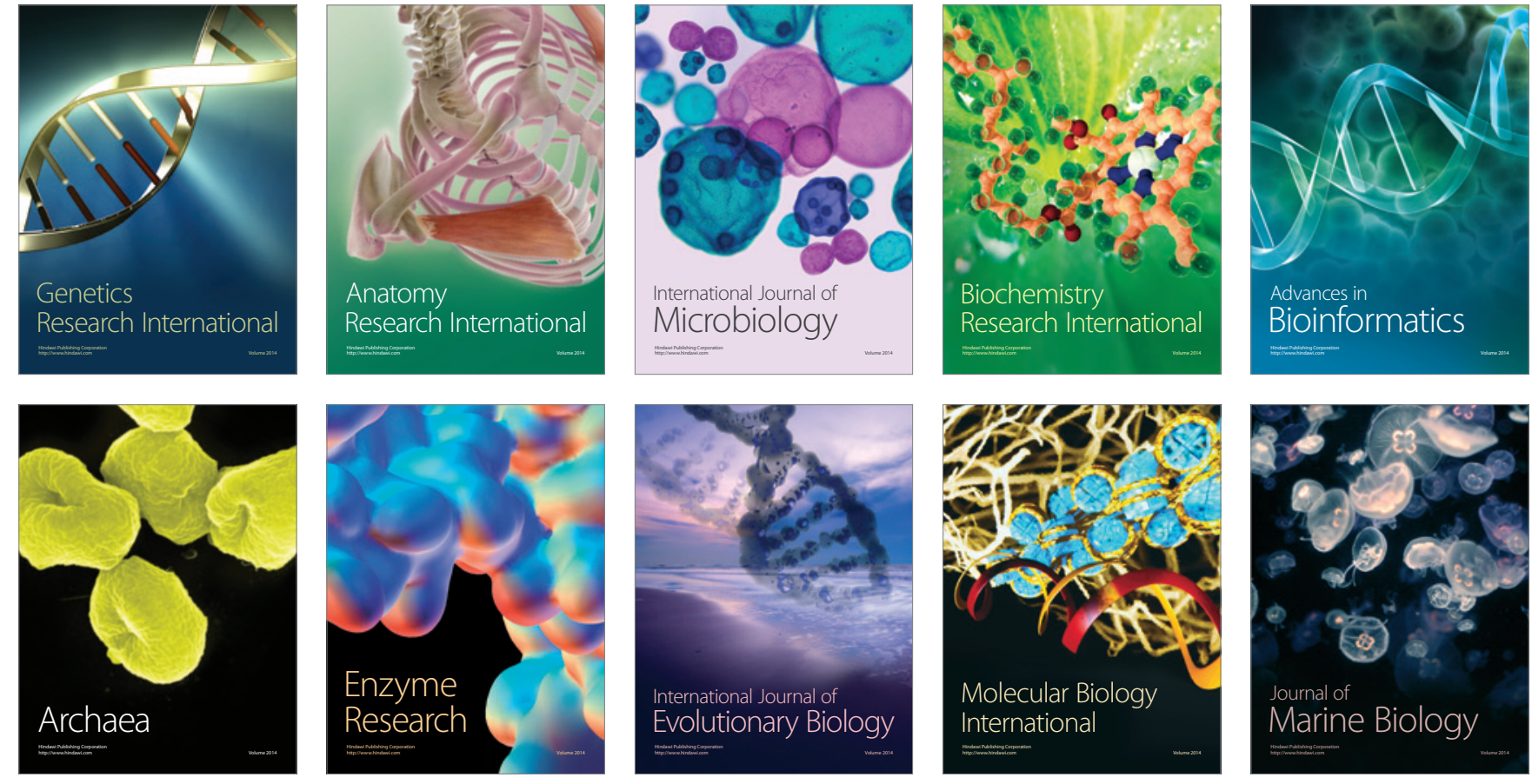